\title{
Short Trajectory Control between Focusing and Tracking Based on Two-Dimensional Equivalent-Perfect Tracking Control for Optical Disk
}

\author{
Takahiro Ohashi* \\ Kiyoshi Ohishi* \\ Student Member, \\ Keisuke Yoshida* \\ Student Member \\ Senior Member, \\ Toshimasa Miyazaki* Member
}

(Manuscript received July 8, 2014, revised Dec. 1, 2014)

\begin{abstract}
A pickup system for optical disks operates in the horizontal and vertical directions for tracking and focusing, respectively. The conventional optical disk system is designed for each direction of operation. The conventional system becomes slow in moving to the next point because it does not consider the operation trajectory. Thus, because the point-to-point movement becomes longer, there is possibility that the tracking performance is deteriorated in highspeed rotation. In order to achieve faster operation, we develop a concurrent control method of tracking and focusing on an optical disk and realize short trajectory control. In this paper, a new two-dimensional feedforward control system is proposed, which has concurrent control of focusing and tracking. The feedforward controller is based on equivalentperfect tracking control (E-PTC). The experimental results show the effectiveness of the proposed control system.
\end{abstract}

Keywords: optical disk, two-dimensional feedforward control system, equivalent-perfect tracking control

\section{Introduction}

The size of digital content is continuously increasing, and therefore, the storage capacity of digital storage media needs to be scaled up correspondingly. Near-field optical recording (NFR) using a solid immersion lens (SIL) was developed to meet these demands. NFR provides a high memory density using a numerical aperture (NA) of the SIL that is greater than 1 and a gap between the disk and lens that is less than $100 \mathrm{~nm}$, which is narrower than the wavelength of the laser beam $^{(1)(2)}$. It is necessary to have a narrow track pitch and gap to achieve a high memory density and large capacity for the optical disk. Therefore, a high-precision focusing and tracking control system are required for optical disks.

It is difficult to design a high-precision focusing and tracking control system because a high-speed optical disk undergoes periodic disturbances induced by the radial run-out and axial eccentricity of the optical disk. Moreover, designing a feedforward controller is difficult because the general optical disk systems do not obtain a reference signal ${ }^{(3)-(5)}$. In order to overcome these problems, we previously has proposed several focusing and tracking control systems, such as a robust feedforward tracking controller based on zero phase error tracking (ZPET) control and prediction of tracking error $^{(6)(7)}$, a ZPET controller with a multi-harmonic disturbance suppression loop ${ }^{(8)}$ and equivalent-perfect tracking control (E-PTC) ${ }^{(9)}$.

Generally, the focusing actuator and the tracking actuator are controlled separately. However, this study realizes concurrent tracking and focusing control for an operation trajectory of the object lens, using a two-dimensional feedforward

\footnotetext{
* Nagaoka University of Technology

1603-1, Kamitomioka-machi, Nagaoka, Niigata 940-2188, Japan
}

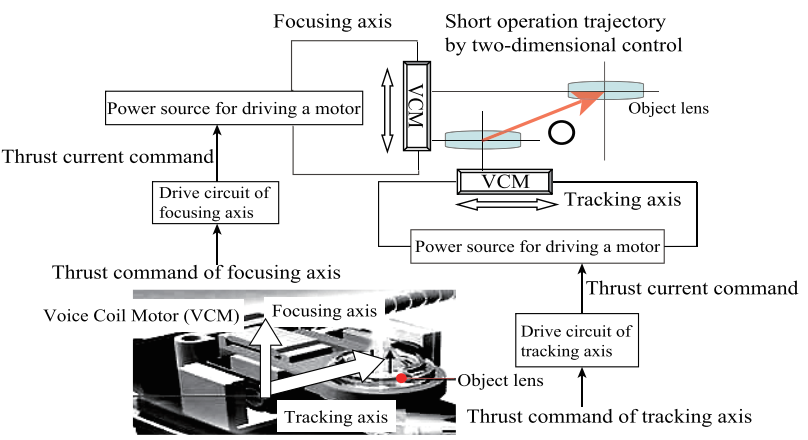

Fig. 1. Strategy of short tracking \& focusing trajectory control

control system. The strategy of the proposed system is introduced for the desired short operation trajectory as shown in Fig. 1. By using the proposed system, it is possible to follow faster than conventional system. Therefore, the tracking performance of the focusing actuator and tracking actuator are improved. The proposed system consists of a feedback controller and two-dimensional feedforward compensators. Each compensator calculates an error vector as a function of the tracking error and the focusing error. The two-dimensional feedforward compensator is controlled by using an error vector. The experimental results in this paper demonstrate the validity of the proposed control system.

\section{Conventional Servo System of Optical Disk System}

2.1 Experimental System In general, the focusing and tracking actuators of the optical disk system use voicecoil motors (VCMs). This paper considers only the first resonant frequency for designing the control system by using second-order plant system models $P_{f}(s)$ and $P_{t}(s)$, which are 


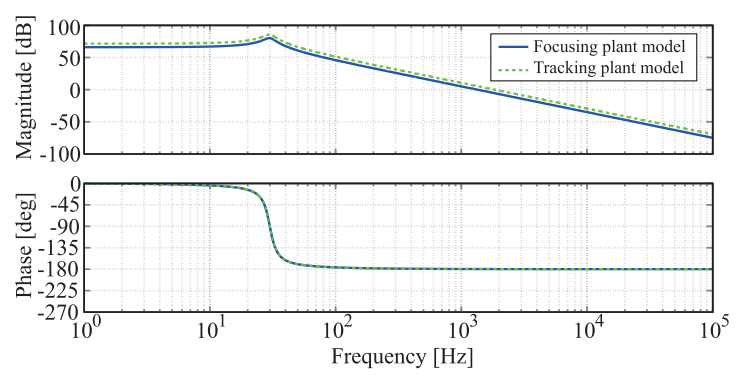

Fig. 2. Frequency characteristics of focusing actuator and tracking actuator

expressed as (1) and (2), respectively. $P_{f}(s)$ is the transfer function of focusing servo system. $P_{t}(s)$ is the transfer function of tracking servo system. The frequency characteristics of the focusing actuator and the tracking actuator are shown in Fig. 2.

$$
\begin{aligned}
& P_{f}(s)=\frac{7.11 \times 10^{7}}{s^{2}+37.3 s+3.55 \times 10^{4}} \\
& P_{t}(s)=\frac{1.58 \times 10^{8}}{s^{2}+37.3 s+3.55 \times 10^{4}}
\end{aligned}
$$

The discrete equation of (1) and (2) are expressed as (3) and (4), respectively.

$$
\begin{aligned}
& P_{f}(z)=\frac{9.87 \times 10^{-3} z+9.87 \times 10^{-3}}{z^{2}-1.99 z+0.99} \\
& P_{t}(z)=\frac{2.19 \times 10^{-2} z+2.19 \times 10^{-2}}{z^{2}-1.99 z+0.99}
\end{aligned}
$$

2.2 E-PTC The perfect tracking control (PTC) ${ }^{(10)-(12)}$ system is a perfect inverse system composed of a multi-rate system. Multi-rate is a particularly effective technique for systems where the sampling frequency is limited. However, as an optical disk system is an analog system, both a singlerate control technology and a multi-rate control technology can be applied. Therefore, the E-PTC system composes the PTC system in a single-rate by using the pseudo inverse matrix.

Figure 3 and Fig. 4 shows the E-PTC system for the foucusing control and the tracking control. The E-PTC is designed using the plant transfer function of second-order system. The state equation and output equation of the controllable canonical form are expressed as in (5) and (6), respectively.

$$
\begin{aligned}
& \mathbf{x}(k+1)=\mathbf{A} \mathbf{x}(k)+\mathbf{B} I(k) \ldots \ldots \ldots \ldots \ldots \ldots \ldots \\
& e(k)=\mathbf{C x}(k)
\end{aligned}
$$

$\mathbf{A}, \mathbf{B}$, and $\mathbf{C}$ are coefficient matrices, $\mathbf{x}(k)$ is the state variable. These coefficient matrices and the state variable are as shown in (7) to (10), respectively. $\mathbf{A}$ is a $\mathrm{n} \times \mathrm{n}$ regular matrix.

$$
\begin{aligned}
\mathbf{A} & =\left[\begin{array}{ccccc}
0 & 1 & 0 & \ldots & 0 \\
0 & 0 & 1 & \ldots & 0 \\
\vdots & \vdots & \vdots & \ddots & \vdots \\
0 & 0 & 0 & \ldots & 1 \\
-a_{0} & -a_{1} & -a_{2} & \ldots & -a_{n-1}
\end{array}\right] \ldots \ldots \ldots \\
\mathbf{B} & =\left[\begin{array}{ccccc}
0 & 0 & \cdots & 0 & 1
\end{array}\right]^{T} \ldots \ldots \ldots \ldots \ldots
\end{aligned}
$$

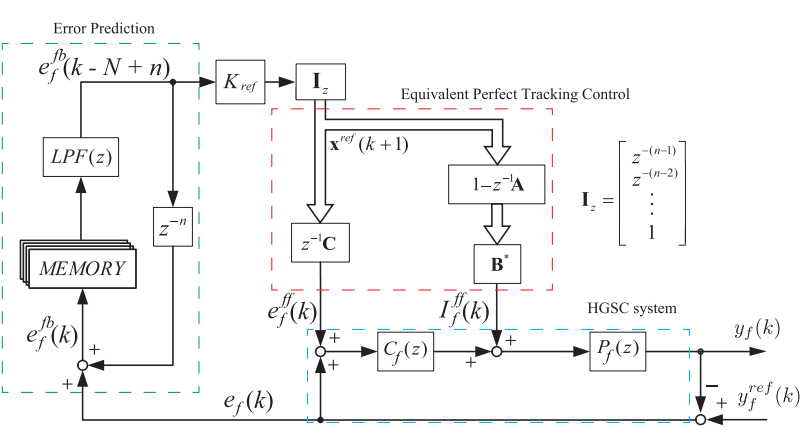

Fig. 3. E-PTC system for focusing control

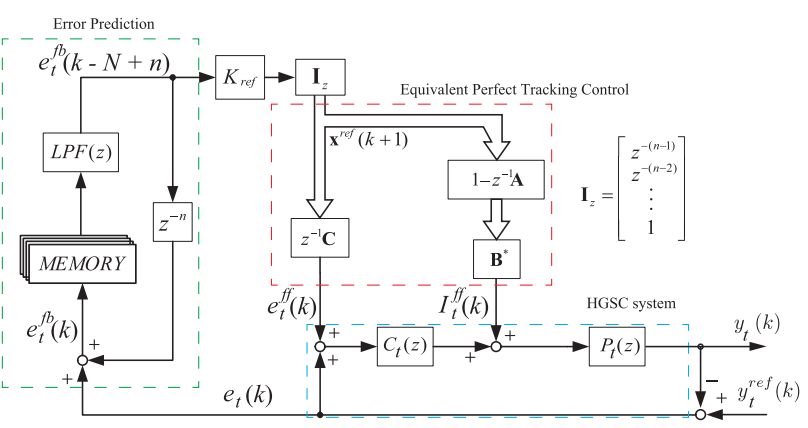

Fig. 4. E-PTC system for tracking control

$$
\begin{aligned}
& \mathbf{C}=\left[\begin{array}{lllll}
c_{0} & c_{1} & \cdots & c_{n-2} & c_{n-1}
\end{array}\right] . \cdots \cdots \\
& \mathbf{x}(k)=\left[\begin{array}{llll}
x(k) & x(k+1) & \cdots & x(k+n-1)
\end{array}\right]^{T} \cdots
\end{aligned}
$$

The inverse matrix of the $\mathbf{B}$ matrix is required to realize the perfect inverse system in (5). However, since the $\mathbf{B}$ matrix is not regular, it is not possible to derive the inverse matrix. Therefore, the E-PTC uses a pseudo-inverse matrix. In this paper, the pseudo-inverse matrix is defined as $\mathbf{B}^{*}$. Thus, the perfect inverse system and the output equation of the E-PTC are expressed as in (11) and (12), respectively.

$$
\begin{aligned}
& I^{f f}(k)=\mathbf{B}^{*}\left(I-z^{-1} \mathbf{A}\right) \mathbf{x}^{r e f}(k+1) \\
& e^{f f}(k)=z^{-1} \mathbf{C x}^{r e f}(k+1) \ldots \ldots
\end{aligned}
$$

In Fig. 3 and Fig. 4, transfer function of feedforward controller are expressed as in (13) and (14), respectively.

$$
\begin{aligned}
E_{f}^{f f}(z) & =K_{r e f} I_{z} \mathbf{B}^{*}\left(1-z^{-1} \mathbf{A}\right) C_{f}^{-1}(z) \\
& +K_{r e f} I_{z} z^{-1} \mathbf{C} \ldots \ldots \ldots \ldots \ldots \\
E_{t}^{f f}(z) & =K_{r e f} I_{z} \mathbf{B}^{*}\left(1-z^{-1} \mathbf{A}\right) C_{t}^{-1}(z) \\
& +K_{r e f} I_{z} z^{-1} \mathbf{C} \ldots \ldots \ldots \ldots \ldots
\end{aligned}
$$

2.3 State Reference Generator The E-PTC requires state reference value $x^{r e f}(k+1)$. Therefore, a state reference generator is designed for the E-PTC using the approximation. The state reference generator is designed from the output equation. If the output equation is expanded, the equation is expressed as in (15).

$$
e(k)=\sum_{j=0}^{n-1} c_{j} \cdot x(k+j)
$$

Here, all state variables are regarded as equal: $x^{r e f}(k)=$ $x(k)=x(k+1)=\cdots=x(k+n-1)$. At this time, it is 
possible to find $K_{\text {ref }}$ as the inverse of $\sum_{j=0}^{n-1} c_{j}$. Further, it is possible to obtain the state reference value $x^{\text {ref }}(k+n)$ from the error value $e^{f b}(k+n)$.

$$
\begin{aligned}
& x^{r e f}(k+n)=\frac{e^{f b}(k+n)}{\sum_{j=0}^{n-1} c_{j}}=K_{r e f} \cdot e^{f b}(k+n) \cdots \cdots \\
& \mathbf{x}^{r e f}(k+1)=\left[z^{-(n-1)} z^{-(n-2)} \cdots 1\right]^{\mathrm{T}} x^{r e f}(k+n) \cdots \cdots
\end{aligned}
$$

$x^{r e f}(k)$ consists of values shifted with respect to each other by one sample. In order to obtain $x^{r e f}(k+1)$, the future value of the tracking error is needed. In the optical disk, the tracking error is periodic. Therefore, the present tracking error is approximated to the error value of the previous cycle, as shown in (18).

$$
e^{f b}(k+n)=e^{f b}(k-N+n) .
$$

$N$ is the number of samples of one rotation.

Now, in order to obtain the $x^{r e f}(k+1), e^{f b}(k)$ is required to shift 2 samples, which is expressed as (19).

$$
e^{f b}(k+2)=e^{f b}(k-N+2) \text {. }
$$

\section{Proposed Two-Dimensional Servo System}

3.1 Error Vector It is necessary to combine the focusing error and tracking error in an optical disk system to achieve concurrent control of focusing and tracking. Usually, the operation directions of focusing actuator and tracking actuator are orthogonal. In this paper, the signals of the focusing error and the tracking error should be considered as being the almost same amplitude. The lissajous curves of the focusing error and the tracking error are shown in Fig. 5. Here, $\theta$ is the phase difference between the focusing error and the tracking error. In Fig. 5, the lissajous curve defines the error vector corresponding to the focusing error and the tracking error.

This paper think that disk is tilted by the disturbance, and the beam spot is moved from A to B. Figure 6 shows the ideal trajectory of the beam spot. In the case of independent control, the laser is set to the tracking after it has set the beam spot of the focusing. On the other hand, because the two-dimensional control have synthesized the focusing and tracking, it want to evenly move the focusing and tracking. In order to this operation, the two-dimensional control is required that the phase difference between the focusing error signal and tracking error signal becomes zero. Thus, the error vector must become like Fig. 5. Therefore, this paper take care of the error signal, and realize the two-dimensional control.

In order to carry out the fine two-dimensional control, the error vector used for focusing control and tracking control is required to have the sine wave characteristics similar to both of the focusing error and the tracking error. At a phase difference $\theta=0^{\circ}$, the lissajous curve is a straight line of error vector. In this case, the phase of the tracking error vector is the same as that of the focusing error vector. Therefore, at $\theta=0^{\circ}$, using the scaling factor, the output amplitude of the tracking control always has the same level as that of the focusing control. This scaling factor is a ratio of the numerator value of (1) and (2). As a result, at $\theta=0^{\circ}$, the twodimensional feedforward control is carried out smoothly and stably.

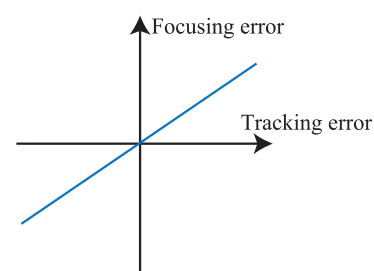

(a) $\theta=0$ [deg]

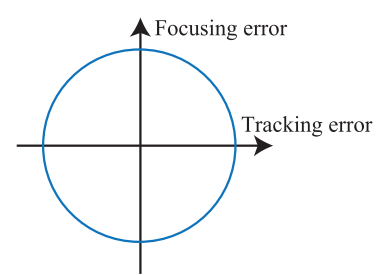

(b) $\theta=90[\mathrm{deg}]$
Fig. 5. Lissajous curves of focusing error and tracking error

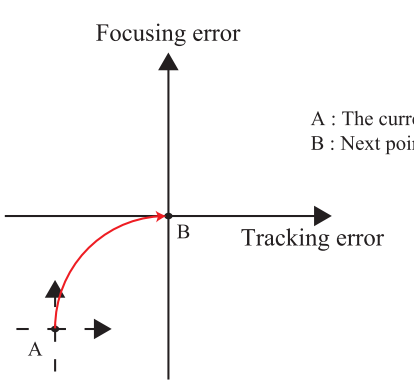

(a) Independent control

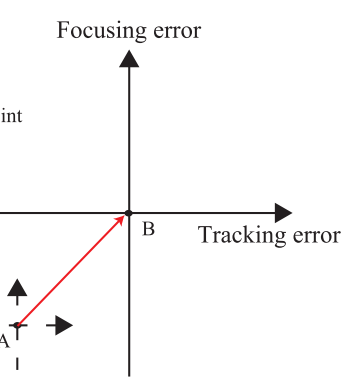

(b) Two-dimensional control
Fig. 6. Movement of the beam spot

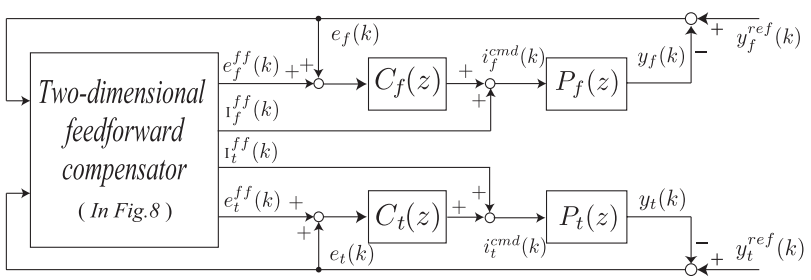

Fig. 7. Two-dimensional feedforward control system

3.2 Design of Feedback Controller Figure 7 shows the two-dimensional control system for focusing and tracking. It consists of feedback controllers and a two-dimensional feedforward compensator. The feedback controllers for focusing and tracking, $C_{f}(z)$ and $C_{t}(z)$, respectively, are presented as in (20) and (21). A feedback controller $C(z)$ is shared between $C_{f}(z)$ and $C_{t}(z)$. A focusing gain $K_{c f}$ and tracking gain $K_{c t}$ are designed to appropriately tune the openloop frequency characteristics.

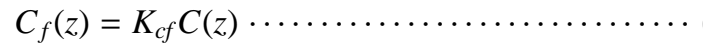

$$
\begin{aligned}
& C_{t}(z)=K_{c t} C(z)
\end{aligned}
$$

In our implementation, the feedback controller $C(s)$ of a continuous-time system is discretized to $C(z)$ by using a zeroorder hold at a sampling frequency $f_{s}$. $C(s)$ consists of phaselag and phase-lead compensators, including an integral element, as expressed in (22).

$$
C(s)=K_{p} \frac{(s-b)(s-c)}{s(s-a)}
$$

In this paper, the $C_{f}(s)$ and $C_{t}(s)$ parameters are designed as follows:

$$
\begin{aligned}
& K_{c f}=0.85, \quad K_{c t}=1.05 \\
& K_{p}=9.36, \quad a=-6.28 \times 10^{4} \\
& b=-5.24 \times 10^{3}+i 3.70 \times 10^{3} \\
& c=-5.24 \times 10^{3}-i 3.70 \times 10^{3}
\end{aligned}
$$

\subsection{Two-Dimensional Feedforward Compensator}

Figure 8 shows a block diagram of the proposed 


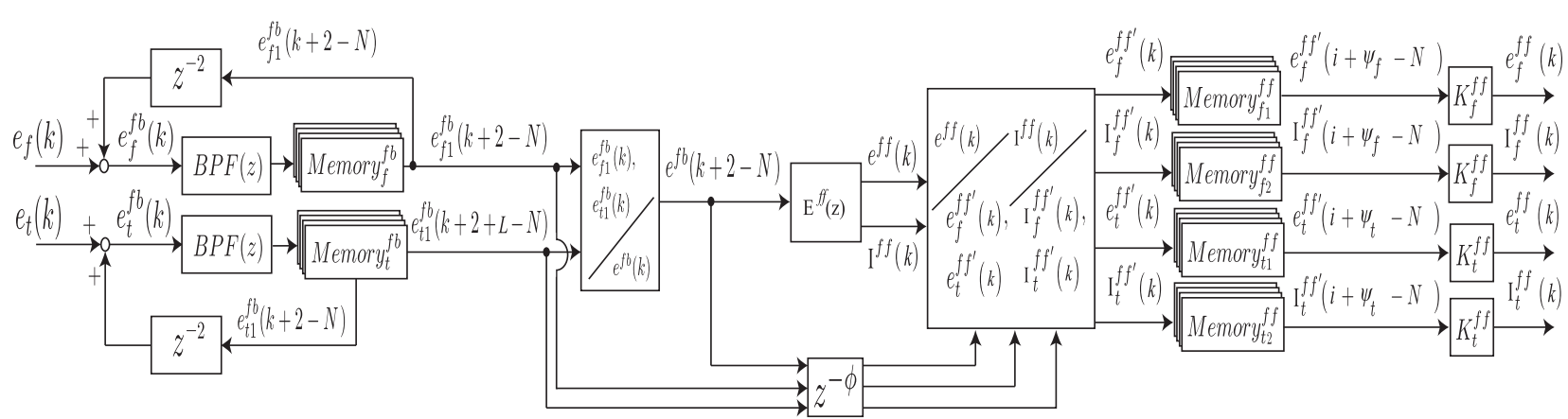

Fig. 8. Two-dementional feedforward compensator

two-dimensional feedforward compensator. Here, $E^{f f}(z)$ is the single feedforward controller based on the E-PTC. $E^{f f}(z)$ is designed by using the imaginary plant system $P_{f t}(s)$ because it is difficult to design a single feedforward controller for the two closed-loop systems for focusing and tracking. From (1) and (2), the denominator polynomials of $P_{f}(s)$ are the same as those of $P_{t}(s)$; therefore, $P_{f t}(s)$ is expressed in (23).

$$
P_{f t}(s) \equiv P_{t}(s)
$$

The discrete equation of $P_{f t}(s)$ is same as $P_{t}(s)$, which is expressed as (24).

$$
P_{f t}(z) \equiv P_{t}(z)=\frac{2.19 \times 10^{-2} z+2.19 \times 10^{-2}}{z^{2}+2 z+1} \cdots \cdots
$$

The feedforward controller $E^{f f}(z)$ is expressed as in (25).

$$
\begin{aligned}
E^{f f}(z)= & K_{r e f} I_{z} \mathbf{B}^{*}\left(1-z^{-1} \mathbf{A}\right) C^{-1}(z) \\
& +K_{r e f} I_{z} z^{-1} \mathbf{C} \ldots \ldots \ldots \ldots
\end{aligned}
$$

$\mathbf{A}$ and $\mathbf{C}$ are presented as in (7) and (9), $\mathbf{B}^{*}$ is the pseudoinverse matrix of (8).

The input variables of the band-pass-filters $B P F(z)$ are the focusing error $e_{f}^{f b}(k)$ and the tracking error $e_{t}^{f b}(k)$. The center frequency of $B P F(s)$ is the disk rotational speed $f_{r}$. Therefore, the fundamental disturbances of $e_{f 1}^{f b}(k)$ and $e_{t 1}^{f b}(k)$ are extracted from $e_{f}^{f b}(k)$ and $e_{t}^{f b}(k)$ using $B P F(z)$, respectively. As mentioned in section 3.1, the error vector at $\theta=0^{\circ}$ is used for the focusing control and tracking control. However, because there is a phase difference $\theta$ between the focusing error and the tracking error, $\theta$ is not always equal to zero. Therefore, the phase difference needs to be reduced to zero by adjusting each detection sampling time. $\theta=0^{\circ}$ is obtained by predicting the $n$-sample futural $e_{t}^{f b}(k)$ by using Memory $y_{t}^{f b}$ in Fig. 8. As a result, the error vector $e^{f b}(k)$ is defined as (26).

$$
e^{f b}(k)=\operatorname{sgn}(k) \sqrt{e_{f 1}^{f b}(k)^{2}+e_{t 1}^{f b}(k+L-N)^{2}} .
$$

$L$ is sample difference of trackig error and focusing error, which is as shown in Fig. 9. Here, a signum function $\operatorname{sgn}(k)$ is expressed as (27).

$$
\operatorname{sgn}(k)=\left\{\begin{array}{rr}
1: & e_{f 1}^{f b}(k) \geq 0 \\
-1: & e_{f 1}^{f b}(k)<0
\end{array}\right.
$$

The relationship between the lassajous curve and the

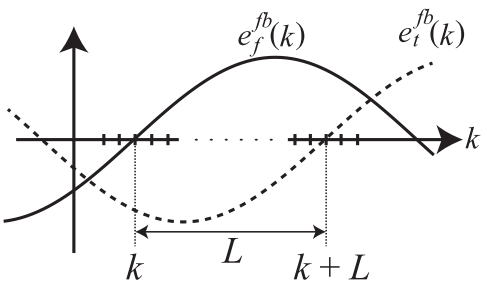

Fig. 9. Zero-cross point difference between focusing error signal and tracking error signal

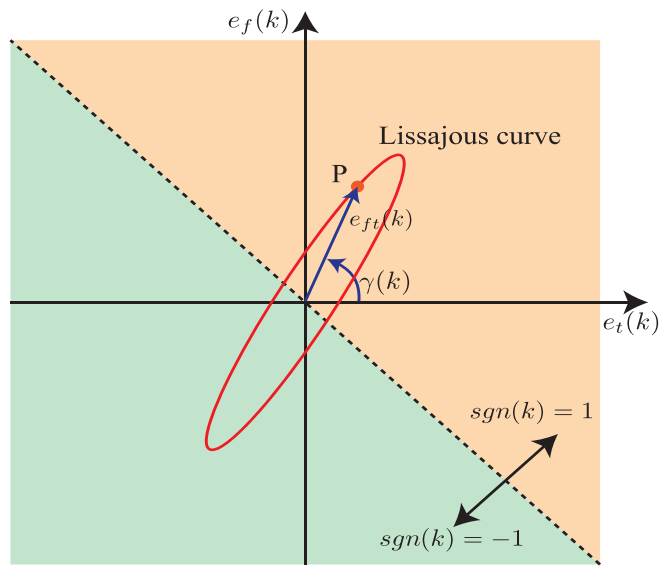

Fig. 10. Definition of sugnum function and lissajous curve

signum function is as shown in Fig. 10. $\gamma(k)$ is the angle of line that connect the point $\mathrm{P}$ and origin, which is derived as shown in (28).

$$
\gamma(k)=\tan ^{-1}\left(\frac{e_{f}(k) / K_{f}}{e_{t}(k) / K_{t}}\right) .
$$

In Fig. 10, $e_{f t}(k)$ is same as $e^{f b}(k)$ of (26). $e^{f b}(k)$ is the feedforward input variable to $E^{f f}(z)$, and $e^{f f}(k)$ and $I^{f f}(k)$ are the feedforward output variable from $E^{f f}(z)$. The feedforward output $e^{f f}(k)$ and $I^{f f}(k)$ are expressed in (11) and (12). $e^{f f}(k)$ and Iff $(k)$ are resolved to $e_{f}^{f f^{\prime}}(k), e_{t}^{f f^{\prime}}(k), I_{f}^{f f^{\prime}}(k)$, and $I_{t}^{f f^{\prime}}(k)$ by using the ratio of $e_{f 1}^{f b}(k)$ and $e_{t 1}^{f b}(k)$. Hence, $e_{f}^{f f^{\prime}}(k), e_{t}^{f f^{\prime}}(k), I_{f}^{f f^{\prime}}(k)$, and $I_{t}^{f^{\prime \prime}}(k)$ are expressed as in (29) to (32), respectively.

$$
\begin{aligned}
e_{f}^{f f^{\prime}}(k) & =e^{f f}(k) \frac{e_{f 1}^{f b}(k+2-N-\phi)}{e^{f b}(k+2-N-\phi)} \cdots \cdots \\
e_{t}^{f f^{\prime}}(k) & =e^{f f}(k) \frac{e_{t 1}^{f b}(k+2+L-N-\phi)}{e^{f b}(k+2+L-N-\phi)} \cdots
\end{aligned}
$$




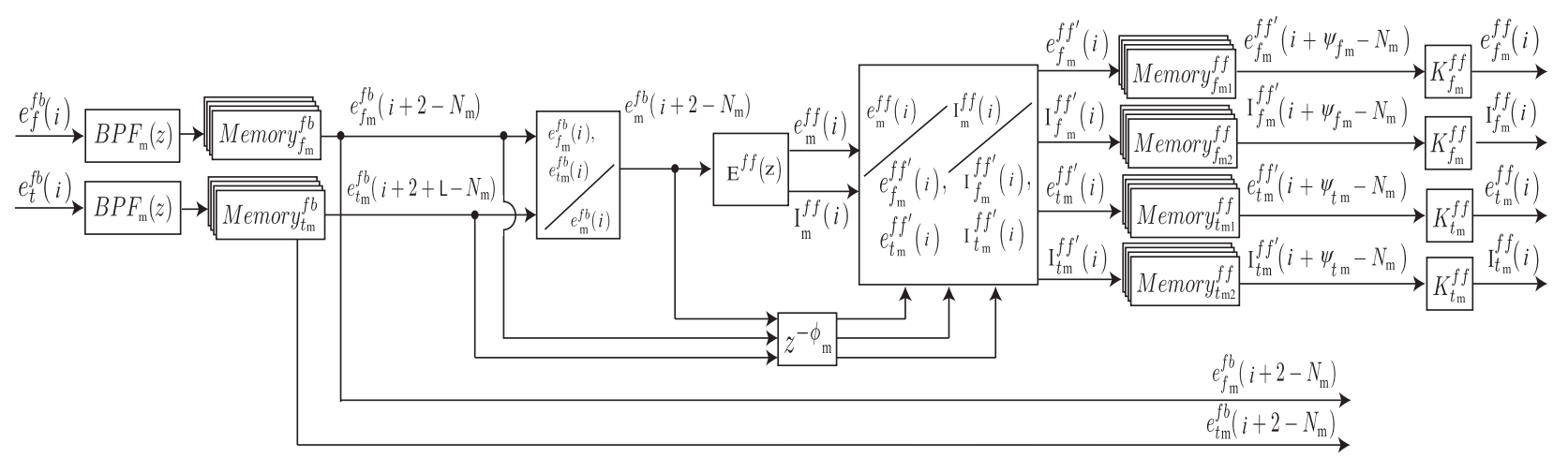

Fig. 11. Feedforward compensator

$$
\begin{aligned}
& I_{f}^{f^{\prime}}(k)=I^{f f}(k) \frac{e_{f 1}^{f b}(k+2-N-\phi)}{e^{f b}(k+2-N-\phi)} \cdots \\
& I_{t}^{f f^{\prime}}(k)=I^{f f}(k) \frac{e_{t 1}^{f b}(k+2+L-N-\phi)}{e^{f b}(k+2+L-N-\phi)}
\end{aligned}
$$

(29) to (32) are shifted 2 samples for obtaining the $x^{r e f}(k+1)$. The details of this mechanism is shown in the section 2.3. Here, $\phi$ is the sampling adjustment value for compensation of the phase-lag of $E^{f f}(z)$ at the disk rotational speed $f_{r}$, which is derived as shown in (33).

$$
\phi=\left\lceil\left.\frac{f_{s}}{f_{r}} \frac{1}{360[\mathrm{deg}]} \angle E^{f f}(z)\right|_{\left.z=e^{j 2 \pi \frac{f_{r}}{f_{s}}}\right\rceil \ldots \ldots \ldots \ldots \ldots . .}\right.
$$

The conventional control system consists of $E_{f}^{f f}(z)$ and $E_{t}^{f f}(z)$ instead of $E^{f f}(z)$. Therefore, it has amplitude error and phase error. These errors are expressed as the output error of feedforward in (34) and (35).

$$
\begin{aligned}
& E_{f}^{\text {diff }}(z)=\frac{E_{f}^{f f}(z)}{E^{f f}(z)} \\
& E_{t}^{\text {diff }}(z)=\frac{E_{t}^{f f}(z)}{E^{f f}(z)}
\end{aligned}
$$

$K_{f}^{f f}$ and $K_{t}^{f f}$ are the scaling amplitude factor as given by (36) and (37), respectively. Further, $\psi_{f}$ and $\psi_{t}$ are the scaling phase factor as given by (38) and (39), respectively. These factors are determined by using (34) and (35).

$$
\begin{aligned}
K_{f}^{f f} & =\left|E_{f}^{d i f f}(z)\right|_{z=e^{j 2 \pi \frac{f_{r}}{f_{s}}}} \cdots \cdots \cdots \cdots \cdots \\
K_{t}^{f f} & =\left|E_{t}^{d i f f}(z)\right|_{z=e^{j 2 \pi \frac{f_{r}}{f_{s}}}} \cdots \cdots \cdots \cdots \cdots \\
\psi_{f} & =\left\lceil\left.\frac{f_{s}}{f_{r}} \frac{1}{360[\mathrm{deg}]} \angle E_{f}^{d i f f}(z)\right|_{z=e^{j 2 \pi} \frac{f_{r}}{f_{s}}}\right\rceil . \\
\psi_{t} & =\left\lceil\left.\frac{f_{s}}{f_{r}} \frac{1}{360[\mathrm{deg}]} \angle E_{t}^{d i f f}(z)\right|_{z=e^{j 2 \pi \frac{f_{r}}{f_{s}}}}\right\rceil .
\end{aligned}
$$

Using from (36) to (39), the final outputs of the twodimensional feedforward controller are $e_{f}^{f f}(k), i_{f}^{f f}(k), e_{t}^{f f}(k)$, and $i_{t}^{f f}$ as shown in (40) to (43), respectively. As the result, the outputs from (40) to (43) become focusing regulation command and tracking regulation command. A $L$-sample past $e_{t}^{f f^{\prime}}(k)$ and $I_{t}^{f^{\prime \prime}}(k)$ are used in (42) and (43) because a $L$-sample futural $e_{t}^{f b}(k)$ is used in (26).

$$
\begin{aligned}
e_{f}^{f f}(k) & =K_{f}^{f f} e_{f}^{f f^{\prime}}\left(k+\psi_{f}-N\right) \cdots \\
I_{f}^{f f}(k) & =K_{f}^{f f} I_{f}^{f^{\prime}}\left(k+\psi_{f}-N\right) \cdots \cdots \\
e_{t}^{f f}(k) & =K_{t}^{f f} e_{t}^{f f^{\prime}}\left(k+\psi_{t}-L-N\right) \\
I_{t}^{f f}(k) & =K_{t}^{f f} I_{t}^{f^{\prime}}\left(k+\psi_{t}-L-N\right)
\end{aligned}
$$

Therefore, the fundamental disturbances are suppressed by the proposed two-dimensional feedforward compensator designed using the E-PTC.

3.4 Multi Feedforward Compensator In the section 3.3, a two-dimensional feedforward compensator is designed to suppress the fundamental disturbances. However, actual optical disk systems also suffer from high-order harmonic disturbances. Therefore, this paper extends the developed two-dimensional feedforward compensator to suppress higher-order harmonic disturbances. Figure 11 shows the feedforward compensator to suppress the mth-order harmonic. Compensator of Fig. 12 is constituted by Fig. 11, which are connected in parellel. This compensator suppresses multi-harmonic disturbances from the fundamental to the mth-order harmonic.

The compensator consists of $m$ two-dimensional feedforward compensators, and requires a large amount of calculation in a digital signal processor (DSP). Hence, it is difficult to realize the control system at a higher sampling frequency $f_{s}\left(=1 / T_{s}\right)$. In order to overcome this problem, the two-dimensional feedforward compensators are discretized at $f_{s} / m\left(=1 / m T_{s}\right)$. As a result, as shown in Fig. 13, the twodimensional feedforward compensators are switched in order with respect to each sample.

\section{Numerical Simulation Results}

In order to comfirm of validity on the tracking control performance and focusing control performance, this paper carries out simulations of the conventional tracking and focusing control systems and the proposed two-dimensional feedforward control system. The numerical simulation conditions are listed in Table 1.

The conventional control system consists of the E-PTC system designed for tracking and focusing, respectively. The conventional feedforward controller and the proposed feedforward compensator have the same control bandwidth of an eighth-order harmonic frequency $(480 \mathrm{~Hz})$. Therefore, the number of two-dimensional feedforward compensators is defined as $m=8$. 


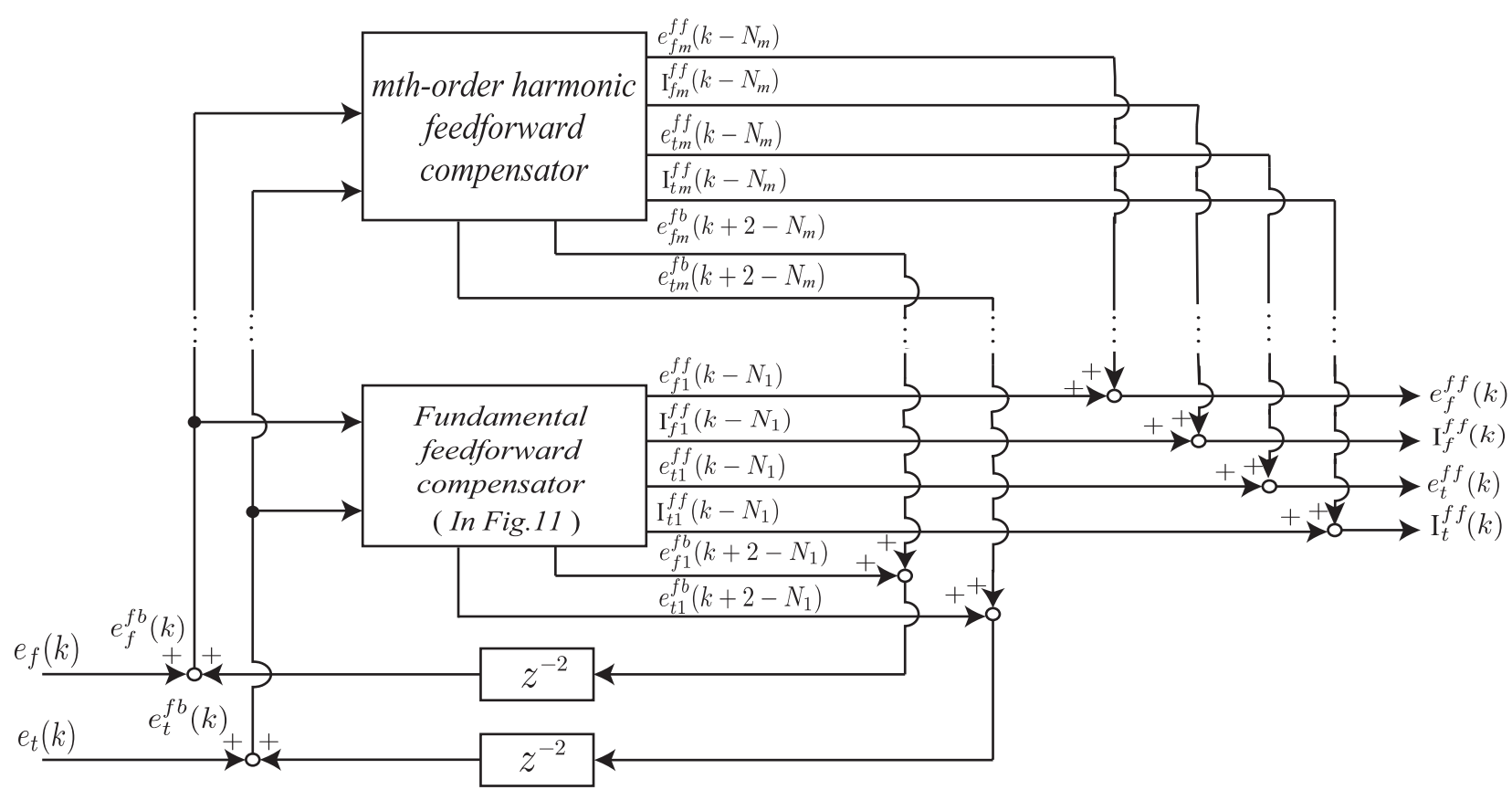

Fig. 12. Multi feedforward compensator based on proposed system

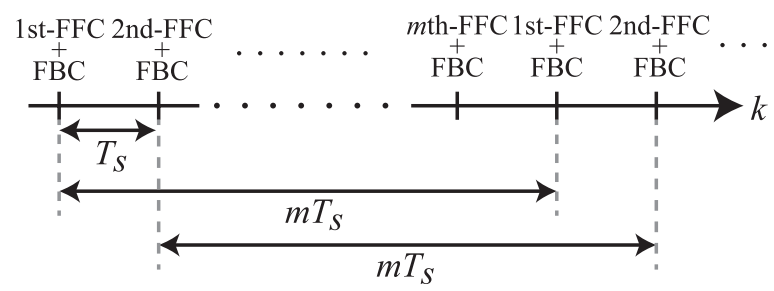

Fig. 13. Sampling periods of feedback controller and feedforward copmensators

Table 1. Experimental conditions

\begin{tabular}{|l|c|}
\hline \hline Rotation speed & $3600 \mathrm{rpm}(60 \mathrm{~Hz})$ \\
\hline Sampling frequency & $60 \mathrm{kHz}$ \\
\hline \hline
\end{tabular}

Table 2. Numerical Simulation Results

\begin{tabular}{c|c|c|c|c|c|}
\hline \hline System & $\begin{array}{c}\text { focus error } \\
{\left[\mathrm{nm}_{\mathrm{p}-\mathrm{p}}\right]}\end{array}$ & $\begin{array}{c}\text { focusing } \\
\pm 3 \sigma[\mathrm{nm}]\end{array}$ & $\begin{array}{c}\text { tracking error } \\
{\left[\mathrm{nm}_{\mathrm{p}-\mathrm{p}}\right]}\end{array}$ & $\begin{array}{c}\text { tracking } \\
\pm 3 \sigma[\mathrm{nm}]\end{array}$ & $\begin{array}{c}\text { length } \\
{[\mathrm{nm}]}\end{array}$ \\
\hline Conventional & 24.48 & \pm 14.88 & 13.60 & \pm 8.44 & 8.60 \\
\hline Proposed & 24.34 & \pm 13.95 & 13.30 & \pm 7.91 & 5.49 \\
\hline \hline
\end{tabular}

Figure 14 shows the numerical simulation results of the conventional system. The $3 \sigma$ value of the focusing error is $14.88 \mathrm{~nm}$, and that of the tracking error is $8.44 \mathrm{~nm}$.

Figure 15 shows the numerical simulation results of the proposed two-dimensional control system. Comparing the proposed system and the conventional system, the $3 \sigma$ value of the focusing error is $12.1 \%$ smaller, at $13.95 \mathrm{~nm}$, and that the tracking error is $6.28 \%$ smaller, at $7.91 \mathrm{~nm}$. The numerical simulation results are listed in Table 2. These results show that the proposed system has a performance equivalent to the conventional system.

Here, the lengths of the lissajous curves are compared for one cycles. The length of lissajous curve is defined by the total from the start point to the end point. Therefore, these lissajous curve contains a maximum value of a one cycle. However, the maximum value of the entire error value does not include. The vertical and horizontal axis of lissajous curves show the focusing error and tracking error. These errors are normalized to $1: 1$. Further, when the lissajous curve of the proposed system is linearly approximated, it is shown in red line. In Fig. 14, the black line lengths of the lissajous curves is $8.60 \mathrm{~nm}$ in the conventional system. In Fig. 15, the black line lengths of the lissajous curves is $5.49 \mathrm{~nm}$ in the proposed system. Thus, the lissajous curve of the proposed system is shortened. In addition, comparing the lissajous curve of the conventional control system and the proposed control system, it is possible to see that the proposed system is linear about $45^{\circ}$ as compared with the conventional control system. The lissajous curve shows that the trajectory of the actuator is short trajectory. The lissajous curve is normalized by the absolute value of each error. The maximum value of the tracking error is on the negative side. Therefore, the positive side becomes smaller.

\section{Experimental Evaluation}

In order to compare the control performance difference between the conventional and the proposed systems, this paper carries out experiments. This section discusses the results of the experiments conducted using a DDU-1000 as a DVD system. The configuration of the experimental device is shown in Fig. 16. The experimental conditions are listed in Table 3.

The conventional feedforward controller and the proposed feedforward compensator have the same control bandwidth of an eighth-order harmonic frequency $(480 \mathrm{~Hz})$. Therefore, the number of two-dimensional feedforward compensators is defined as $m=8$.

Figure 17 shows the experimental results of the conventional system. The $3 \sigma$ value of the focusing error is $24.29 \mathrm{~nm}$, and that of the tracking error is $4.62 \mathrm{~nm}$.

Figure 18 shows the experimental results of the proposed two-dimensional control system. The $3 \sigma$ value of the focusing error is $29.1 \%$ smaller, at $17.22 \mathrm{~nm}$, and that the tracking 


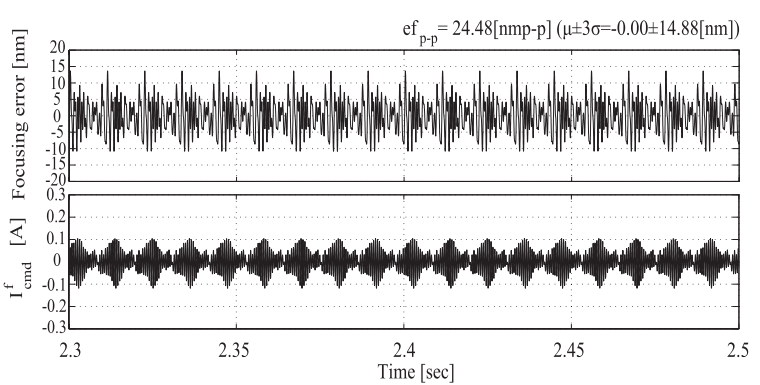

(a) Focusing error signal and focusing command current

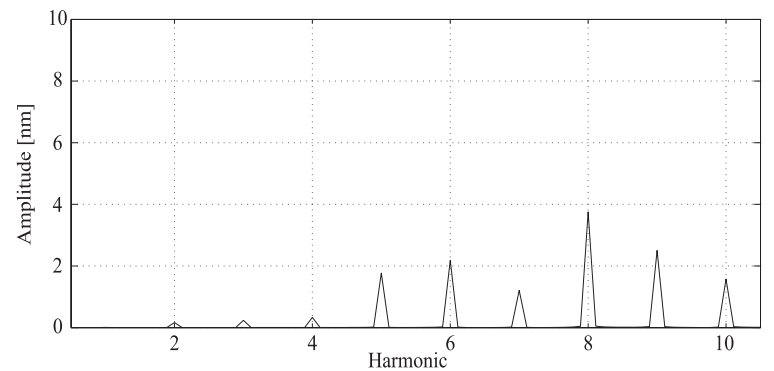

(b) FFT results of focusing error signal

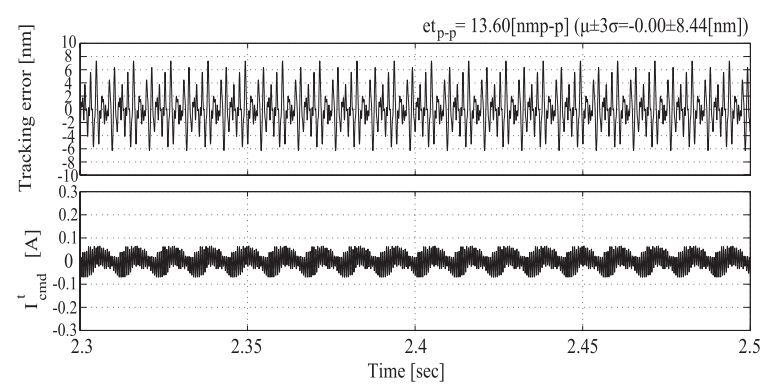

(c) Tracking error signal and tracking command current

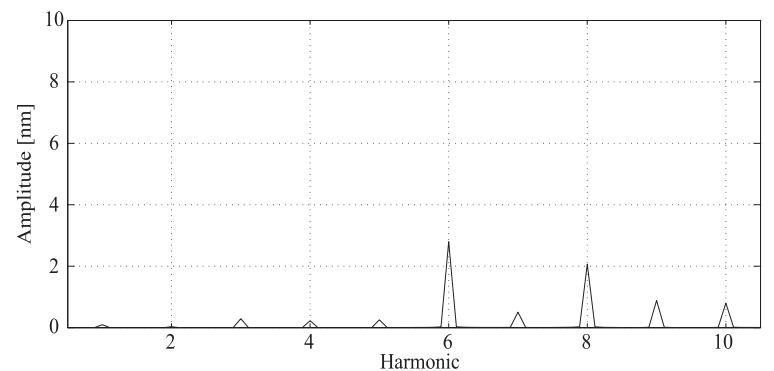

(d) FFT results of tracking error signal

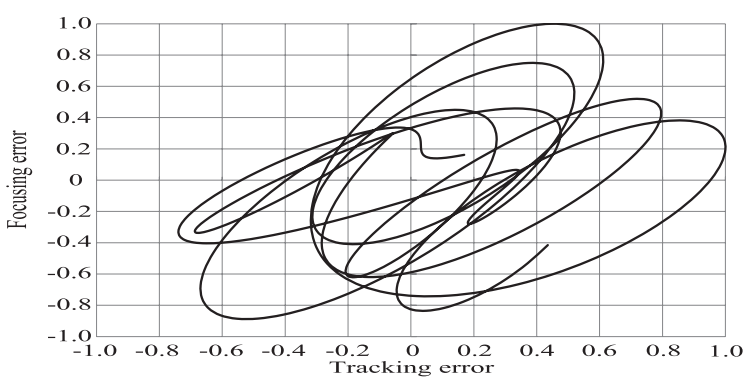

(e) Lissajous curve of proposed system

Fig. 14. Simulation results of conventional system

error is $43.3 \%$ smaller, at $2.62 \mathrm{~nm}$. Table 4 summarizes the experimental results. The reduction in $3 \sigma$ indicates that the performance of the control system is improved.

As with the simulation result, the lissajous curve of the proposed control system is linear as compared with the conventional control system. In Fig. 17, the black line length

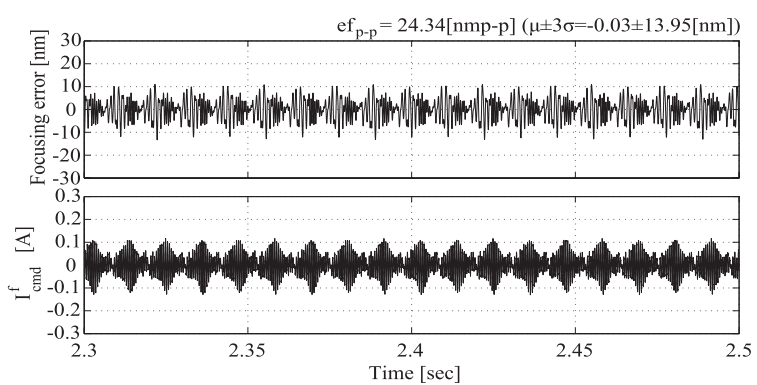

(a) Focusing error signal and focusing command current

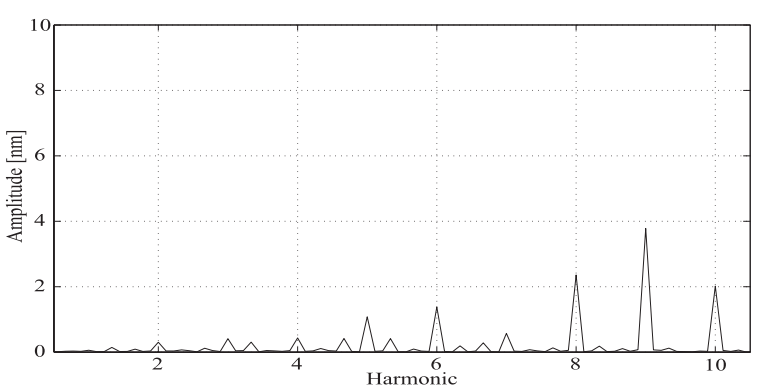

(b) FFT results of focusing error signal

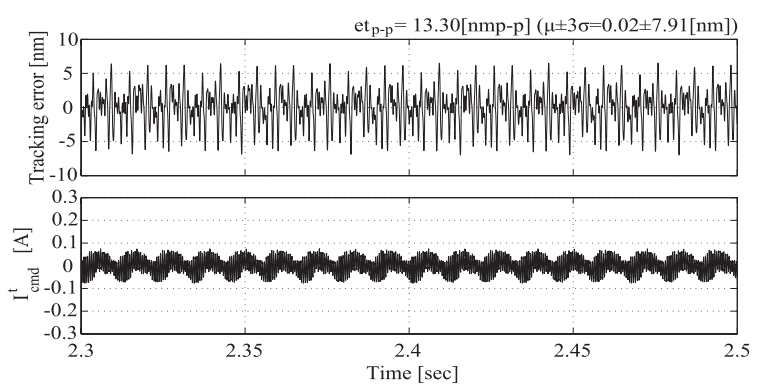

(c) Tracking error signal and tracking command current

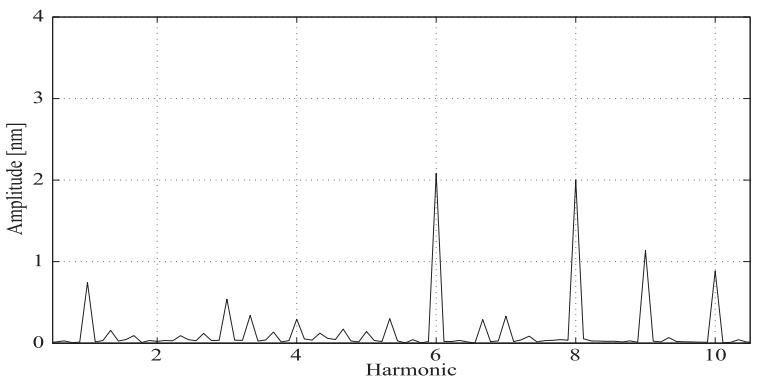

(d) FFT results of tracking error signal

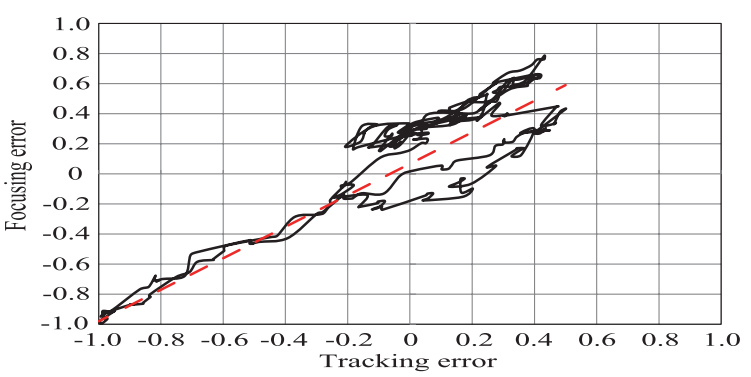

(e) Lissajous curve of proposed system

Fig. 15. Simulation results of proposed system

of the lissajous curve is $6.98 \mathrm{~nm}$ in the conventional system. In Fig. 18, the black line length of the lissajous curve is $4.28 \mathrm{~nm}$ in the proposed system. This short lissajous curve indicate that the the focusing and tracking performances are improved. Thus, the control performance of the proposed 


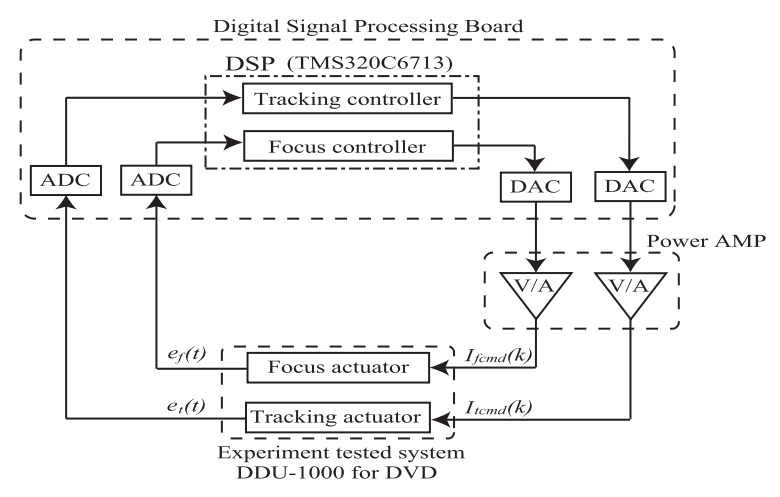

Fig. 16. Configuration of experimental device

Table 3. Experimental conditions

\begin{tabular}{|l|c|}
\hline \hline Wavelength & $405 \mathrm{~nm}$ \\
\hline NA & 0.65 \\
\hline Media type & DVD+R \\
\hline Track pitch & $0.74 \mu \mathrm{m}$ \\
\hline Rotation speed & $3600 \mathrm{rpm}(60 \mathrm{~Hz})$ \\
\hline Sampling frequency & $60 \mathrm{kHz}$ \\
\hline DSP & TMS320C6713 \\
\hline \hline
\end{tabular}

Table 4. Experimental Results

\begin{tabular}{|c|c|c|c|c|c|}
\hline \hline System & $\begin{array}{c}\text { focus error } \\
{\left[\mathrm{nm}_{\mathrm{p}-\mathrm{p}}\right]}\end{array}$ & $\begin{array}{c}\text { focusing } \\
\pm 3 \sigma[\mathrm{nm}]\end{array}$ & $\begin{array}{c}\text { tracking error } \\
{\left[\mathrm{nm}_{\mathrm{p}-\mathrm{p}}\right]}\end{array}$ & $\begin{array}{c}\text { tracking } \\
\pm 3 \sigma[\mathrm{nm}]\end{array}$ & $\begin{array}{c}\text { length } \\
{[\mathrm{nm}]}\end{array}$ \\
\hline Conventional & 33.85 & \pm 24.29 & 6.65 & \pm 4.62 & 6.98 \\
\hline Proposed & 28.29 & \pm 17.22 & 5.39 & \pm 2.62 & 4.28 \\
\hline
\end{tabular}

system is improved because the operation trajectory is shorter. Therefore, the proposed control system achieves better high-precision focusing and tracking performance than the conventional control system.

\section{Conclusion}

This paper proposes a two-dimensional feedforward control system based on E-PTC. The proposed control system consists of a feedback controller and multiple twodimensional feedforward compensators. The error vectors at each harmonic are a combination of the focusing error and tracking error of the multiple compensators. Each error vector is regulated by the feedforward controller on the basis of the E-PTC. Experimental results confirm that the proposed control system exhibits good focusing and tracking performance. In addition, the control performance is improved by the shorter operation trajectory.

\section{References}

(1) I. Ichimura, K. Kishima, K. Osato, K. Yamamoto, Y. Kuroda, and K. Saito: "Near-Field Phase-Change Optical Recording of 1.36 Numerical Aperture", Jpn. J. Appl. Phys., Vol.39, No.2, pp.962-967 (2000)

( 2 ) T. Ishimoto, A. Nakaoki, K. Saito, T. Yamasaki, T. Yukumoto, S. Kim, T. Kondo, T. Mizukuki, O. Kawakubo, M. Honda, N. Shinohara, and N. Saito: "High-Density Recording with a Near-Field Optical Disk System Using a Medium with a Top Layer of High Refractive Index", Jpn. J. Appl. Phys., Vol.48, 03 A015 (2009)

( 3 ) T. Yamaguchi, H. Numasato, and H. Hirai: "A Mode-switching Control for Motion Control and its Application to Disk drives: Design of Optical Modeswithcing Condition", IEEE/ASME Trans. on Mechatronics, Vol.3, No.3, pp.202-209 (1998)

( 4 ) A. Okuyama, M. Soma, T. Yoshida, N. Yanagihara, Y. Mii, and H. Yamada: "Robust Control of CD-ROM Drives Using Multirate Disturbance Observer",

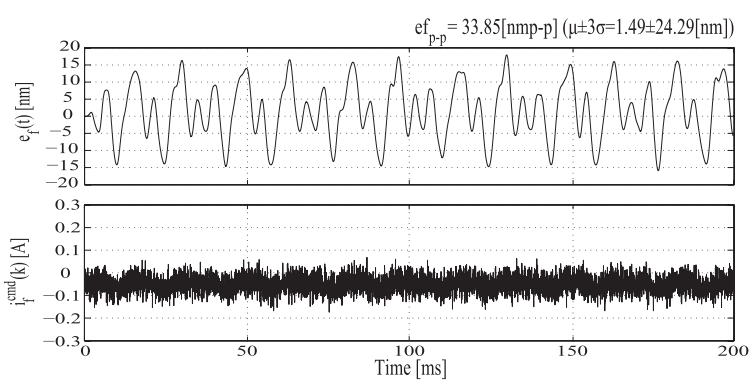

(a) Focusing error signal and focusing command current

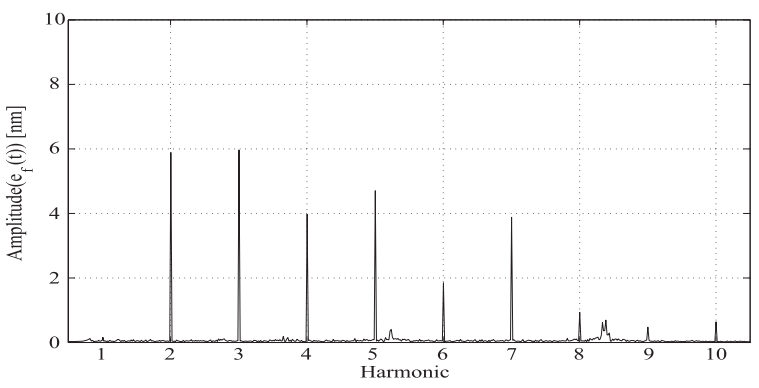

(b) FFT results of focusing error signal
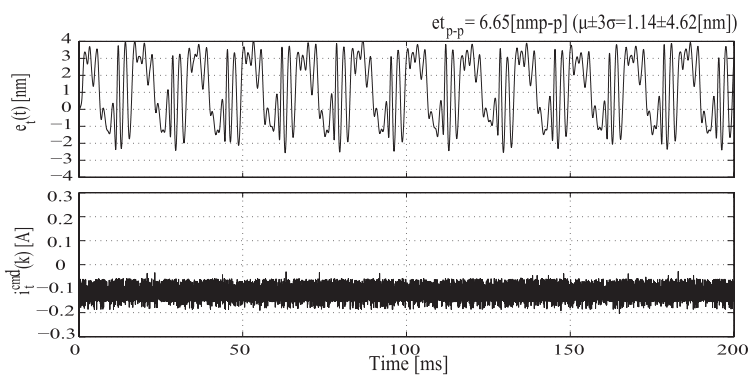

(c) Tracking error signal and tracking command current

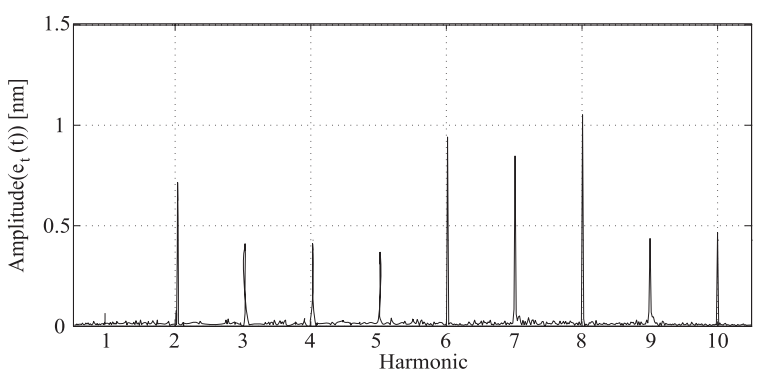

(d) FFT results of tracking error signal

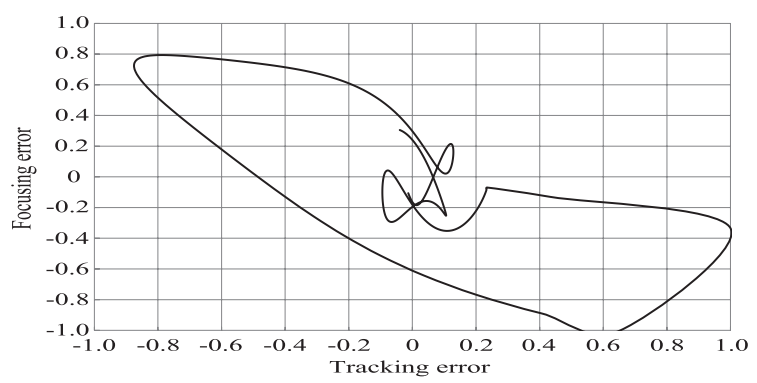

(e) Lissajous curve of proposed system

Fig. 17. Experimental results of conventional system

JSME International Journal, Vol.41, No.4, pp.806-812 (1998-12)

( 5 ) K. Yang, Y. Choi, and W.K. Chung: "On the Tracking Performance Improvement of Optical Disk Drive Servo Systems Using Error-Based Disturbance Observer", IEEE Transactions on Industrial Electronics, Vol.52, No.1 (20052)

( 6 ) D. Koide, H. Yanagisawa, H. Tokumaru, S. Nakamura: K. Ohishi, K. Inomata, and T. Miyazaki: "High-speed Tracking Method using ZPET-FF Control for High-Data-Rate Optical Disk Drives", Tech. Digest of ISOM 


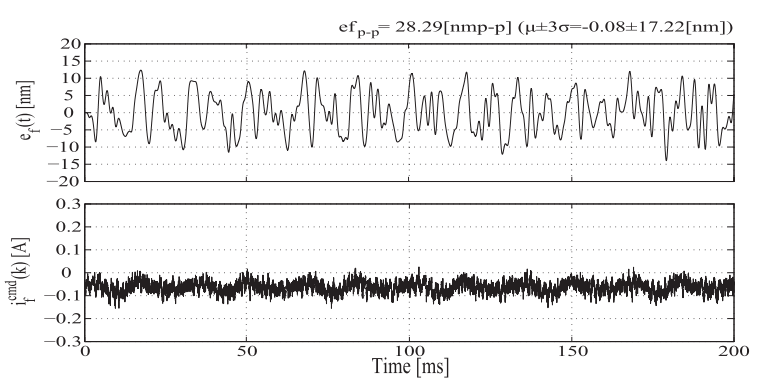

(a) Focusing error signal and focusing command current

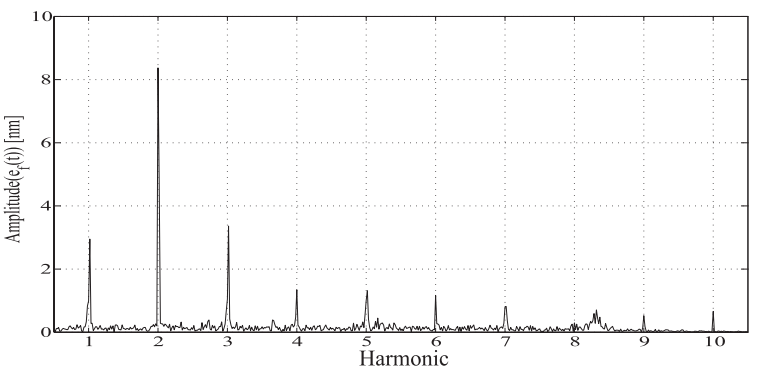

(b) FFT results of focusing error signal

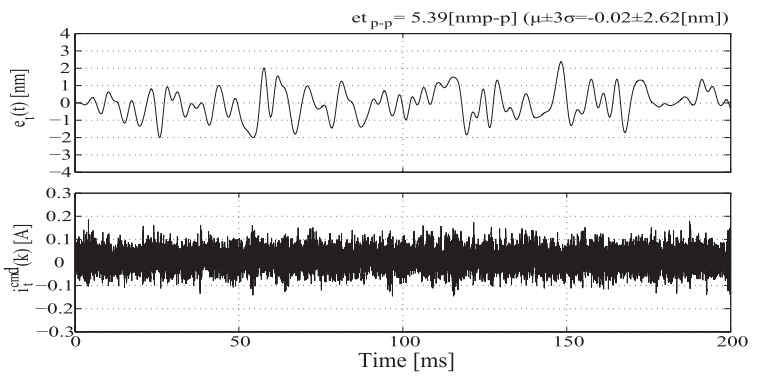

(c) Tracking error signal and tracking command current

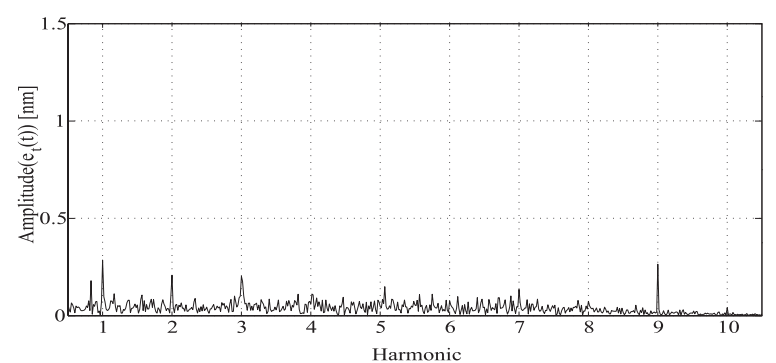

(d) FFT results of tracking error signal

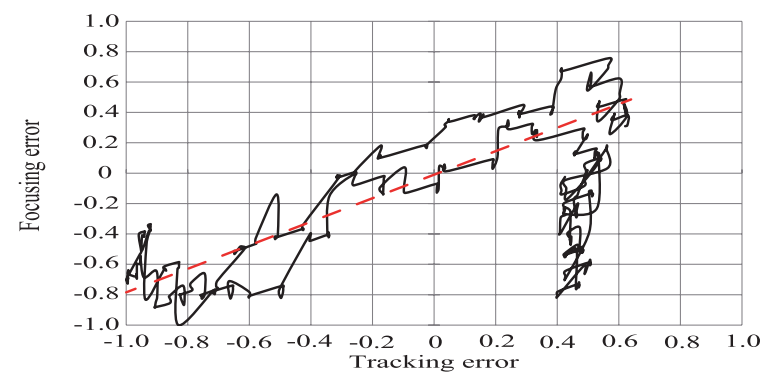

(e) Lissajous curve of proposed system

Fig. 18. Experimental results of proposed system

2003, pp.40-41 (2003)

( 7 ) M. Tomizuka: "Zero Phase Error Tracking Algorithm for Digital Control", ASME Journal of Dynamic Systems, Measurement and Control, Vol.113, pp.6-10 (1987)

( 8 ) T. Nakazaki, T. Ogata, K. Ohishi, T. Miyazaki, M. Sazawa, D. Koide, Y. Takano, and H. Tokumaru: "Focusing Control System for Suppressing HighOrder Harmonic Disturbances in Optical Disk Systems", IIC-12-15, pp.83$88(2012-3)$

(9) T. Ogata, N. Sakimura, T. Nakazaki, K. Ohishi, T. Miyazaki, D. Koide, H. Tokumaru, and Y. Takano: "Tracking Control System with Equivalent Perfect Tracking Control for Optical Disk", IEEJ Transactions on Industry Applications, Vol.132, No.12, pp.1121-1130 (2012)

(10) H. Fujimoto, Y. Hori, and A. Kawamura: "Perfect tracking control based on multirate feedforward control with generalized sampling periods", IEEE Trans. Ind. Electron, Vol.48, No.3, pp.636-344 (2001)

(11) H. Fujimoto and Y. Hori: "RRO Compensation of Hard Disk Drives With Multirate Repetitive Perfect Tracking Control", IEEE Trans. Ind. Electron, Vol.56, No.10, pp.3825-3831 (2009)

(12) H. Fujimoto, Y. Hori, T. Yamaguchi, and S. Nakagawa: "Proposal of seeking control of hard disk drives based on perfect tracking control using multirate feedforward control", Proc. IEEE AMC, pp.74-79 (2000)

(13) T. Ogata, Y. Nabata, K. Ohishi, T. Miyazaki, M. Sazawa, D. Koide, Y. Takano, and H. Tokumaru: "Perfect Tracking Control System with Prediction State Observer for Next Generation Optical Disk", Tech. Digest of ISOM2010, Mo-C-03 (2008)

Takahiro Ohashi (Student Member) received B.S. degree in Electri-

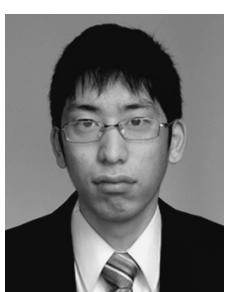
cal, Electronics and Information Engineering from Nagaoka University of Technology, Japan in 2013. Now he is a candidate of the M.S. degree in Electrical, Electronics and Information Engineering. His research interests include servo system of optical disk. $\mathrm{He}$ is a student member of the Institute of Electrical Engineers of Japan (IEEJ).

Keisuke Yoshida

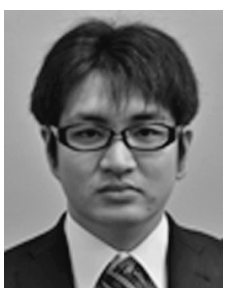

(Student Member) received B.S. degree in Electrical, Electronics and Information Engineering from Nagaoka University of Technology, Japan in 2014. Now he is a candidate of the M.S. degree in Electrical, Electronics and Information Engineering. His research interests include servo system of optical disk. $\mathrm{He}$ is a student member of the Institute of Electrical Engineers of Japan (IEEJ).

Kiyoshi Ohishi (Senior Member) received the B.S., M.S., and Ph.D.

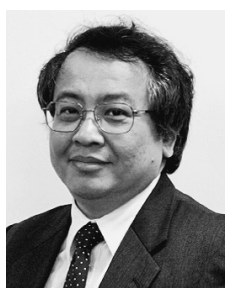
degrees, all in electrical engineering, from Keio University, Yokohama, Japan, in 1981, 1983, and 1986, respectively. From 1986 to 1993, he was an Associate Professor with Osaka Institute of Technology, Osaka, Japan. From 1993 to 2003, he was an Associate Professor with Nagaoka University of Technology, Niigata, Japan. Since August 2003, he has been a Professor at the same university. He is an administration committee member of the IEEE Industrial Electronics Society, the Institute of Electrical Engineers of Japan (IEEJ), the Japan Society of Mechanical Engineers (JSME), the Society of Instrument and Control Engineers (SICE), and the Robotics Society of Japan (RSJ).

Toshimasa Miyazaki (Member) received the B.S., M.S., and Ph.D.

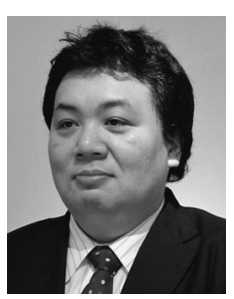
degrees, all in electrical engineering, from Nagaoka University of Technology, Niigata, Japan, in 1994, 1996, and 1999, respectively. From 1999 to 2009, he was an Associate Professor with Nagaoka National College of Technology, Niigata, Japan. Since 2010, he has been an Associate Professor with Nagaoka University of Technology, Niigata, Japan. His research interests include motion control and power electronics. He is a member of the IEEE Industrial Electronics Society, the Institute of Electrical Engineers of Japan (IEEJ), and the Society of Instrument and Control Engineers (SICE). 